\title{
Low Mast Cell Density Predicts Poor Prognosis in Oral Squamous Cell Carcinoma and Reduces Survival in Head and Neck Squamous Cell Carcinoma
}

\author{
CECILIE GJØVAAG ATTRAMADAL ${ }^{1}$, SHEEBA KUMAR ${ }^{1}$, JIAN GAO $^{1}$, MORTEN EBBE BOYSEN ${ }^{2}$, \\ TROND SUNDBY HALSTENSEN ${ }^{1,3}$ and MAGNE BRYNE ${ }^{1}$ \\ ${ }^{1}$ Department of Oral Biology, Faculty of Dentistry, University of Oslo, Oslo, Norway; \\ ${ }^{2}$ Department for Otorhinolaryngology, Head and Neck Surgery, \\ Oslo University Hospital, Rikshospitalet, Oslo, Norway; \\ ${ }^{3}$ Department of Medicine, Lovisenberg Diakonale Hospital, Oslo, Norway
}

\begin{abstract}
Background: The cellular composition of the tumor microenvironment (TME) at the invading front of oral squamous cell carcinomas (OSCCs) may reflect biologically important cancer features and host responses, and thus be related to disease progression. The TME density of mast cells (MCs), macrophages, cancer-associated fibroblasts (CAFs) and endothelial cells were quantified at the invasive front and analyzed regarding their relation to disease recurrence in patients with small T1/2NOMO OSCCs. mRNA for MC-specific proteins were analyzed in a second patient cohort with head and neck squamous cell carcinoma (HNSCC). Materials and Methods: Samples from 62 patients with T1/2NOMO OSCC were immunohistochemically stained and scored for the cellular expression of mast/stem cell growth factor receptor (c-KIT) (MCs), CD68 (macrophages), $\alpha$-smooth muscle actin ( $\alpha$-SMA) (CAFs) and CD31 (endothelial cells) and this was analyzed according to disease recurrence. Data from The Cancer Genome Atlas database were used to examine mRNA expression profiles and clinical data of patients with 399 HNSCC. Results: Increased MC density at the invasive front was significantly associated with reduced disease recurrence, as none of the patients with high MC density experienced relapse. Moreover, increased expression of $m R N A$ for $M C$
\end{abstract}

This article is freely accessible online.

Correspondence to: Cecilie Gjøvaag Attramadal, Department of Oral Biology, Faculty of Dentistry, University of Oslo, PB 1052 Blindern, 0316 Oslo, Norway. E-mail: c.g.attramadal@odont.uio.no and Magne Bryne, Department of Oral Biology, Faculty of Dentistry, University of Oslo, PB 1052 Blindern, 0316 Oslo, Norway. E-mail: magnebr@odont.uio.no

Key Words: Head and neck squamous cell carcinoma, mast cell, prognosis, invasive front. specific markers as $c$-KIT, and $\alpha$-, $\beta$-, and $\delta$-tryptases and the $M C$-stimulating factor, stem cell factor (SCF), was significantly associated with good prognosis in patients with HNSCC. Conclusion: Decreased MC density at the invasive front may reflect tumor biology related to disease progression and prognosis. Counting MCs seems to be an easy and practical tool, that could be utilized for prognostic evaluation.

The growth of oral squamous cell carcinoma cells is influenced by its stroma. Stromal cells such as mast cells (MCs), cancer-associated fibroblasts (CAFs), tumorassociated macrophages (TAMs), endothelial cells, pericytes, smooth muscle cells and lymphocytes promote tumor growth, local infiltration, proliferation, neovascularization and escape from immune defense (1). Such cancer-amended stroma is referred to as the tumor microenvironment (TME) (2), and is probably as important as the cancer itself in facilitating cancer cell invasion and metastasis. In the current study of early-stage oral squamous cell carcinomas (OSCCs), we examined the significance of MCs, CAFs, TAMs and endothelial cells in the TME of the invasive front, and their influence on disease recurrence. The MC findings were further explored in a larger cohort of patients with head and neck squamous cell carcinoma.

MCs are especially present in surface mucosa such as the skin, bronchial tract, lungs and digestive tract, conjunctiva, nose and mouth, and are best known for their role in allergic immune reactions (3). The TME in OSCC contains more MCs than normal oral mucosa (4), but their role in TME is intricate and poorly understood. These MCs may promote cancer growth by stimulating angiogenesis and tissue remodeling, but also exert anticancerous effects, depending on cofactors and cell location (5).

CAFs represent a common functional state of cancerstimulated mesenchymal cells. Their cell of origin is 
unknown, but they may arise from stem cells, epithelialmesenchymal transition (EMT)-transformed cancer cells, fibroblasts or even pericytes. CAFs may promote cancer infiltrative growth, stimulate angiogenesis and modulate immune responses (6).

TAMs may also promote tumor growth, as a high density of TAMs seems to be associated with increased mortality in patients with gastric, urogenital and mammary cancer, and $\operatorname{OSCC}(7,8)$.

Tumors larger than 1-2 mm become hypoxic and nutrient deprived if no angiogenesis occurs (9). Cancer cells release soluble growth factors, chemokines and cytokines, which initiate sprouting and proliferation of quiescent endothelial cells of nearby blood vessels and lymphatics (9). These signals also recruit fibroblasts that produce extracellular matrix. Proliferation and metastasis require vascularization and high angiogenic stimulation, which may be visualized by high endothelial cell density in TME (9).

The aim of this study was to examine if the cellular composition in the infiltrative front influenced disease recurrence of small OSCCs.

\section{Materials and Methods}

\section{Patients with OSCC}

Patients and tumors. Relevant clinical information, follow-up and cause of death for all patients (with no history of previous head and neck cancer) were recorded prospectively at the Department of Otolaryngology, Oslo University Hospital, Norway, in collaboration with The Norwegian Radium Hospital, over a period of 15 years (1983-1997). One of the Authors (MEB) has an approved authorization from The Norwegian Data Inspectorate and the Ministry of Health to collect and analyze data from patients with head and neck cancer admitted to the Oslo University Hospital. The Regional Ethical Committee approved the study (2013/1178S05045). Classification was initially carried out according to the UICC, third edition (1982), and later updated to new editions. The database includes 392 patients with T1-2/N0, M0 OSCC (lip and base of tongue tumors were excluded) treated with curative intent. Adequate tumor tissue for further examination was available in only 62 patients, 22 females and 40 males. Fifteen patients had recurrent disease. Tumor sites were: gingiva $(\mathrm{N}=13)$, tongue $(\mathrm{N}=17)$, floor of mouth $(\mathrm{N}=25)$ and other sites $(\mathrm{N}=7)$ (buccal mucosa, palate and unspecified mouth region). There were $29 \mathrm{~T} 1$ and $33 \mathrm{~T} 2$ tumors. Eleven patients were 50 years old or younger, 14 were aged between 51 and 60 years, and 37 were older than 60 years.

Treatment. The patients were treated with initial surgical excision. All operative specimens were subjected to meticulous histopathological examination. An excision was considered complete when at least three high-power microscopic fields were free of tumor cells, and 39 patients received postoperative radiotherapy due to suspicion regarding the excision completeness. Pre-treatment biopsies were examined and used for two patients who received preoperative radiotherapy. External radiotherapy was given using a high-voltage source, and the primary site received sequential 2 Gy local treatments/day, 5 days a week, for a total of 60-70 Gy, and the neck received 50-60 Gy at levels I-VI.

Follow-up. The follow-up time (mean=55 months, range=3-151 months) was measured from the time of cancer diagnosis. Complete follow-up information, including disease recurrence and cause of death, was retrieved from the outpatient and hospital records, autopsy reports, family physicians, local hospitals or by interviews with the patients themselves or next of kin. Fifteen patients developed a recurrent disease during the follow-up period.

Histopathology. Formalin fixed, paraffin-embedded archival material was processed according to standard procedures for hematoxylin-eosin (HE) staining. As described previously (10), a pathologist (Hari P. Dhakal) verified OSCC diagnosis and graded the tumors in a blinded fashion according to the WHO classification, and the invasive front grading (IFG) system, by taking cellular pleomorphism, keratinization, host response and pattern of invasion into account (10).

Immunohistochemistry (IHC). Tumor sections (formalin-fixed, paraffin-embedded, and 3 to $5 \mu \mathrm{m}$-thick) were pretreated in PT Link (a procedure combining deparaffinizing and heat-induced epitope retrieval without xylol). The slides were then immersed in a preheated $\left(65^{\circ} \mathrm{C}\right)$ buffer (Flex Target Retrieval Solution, Dako Cytomation Denmark A/S, Glostrup, Denmark), gradually heated to $97^{\circ} \mathrm{C}, 20 \mathrm{~min}$ at $97^{\circ} \mathrm{C}$, and then gradually $(60-70 \mathrm{~min})$ cooled to $65^{\circ} \mathrm{C}$, before being washed in Dako Wash Buffer (Dako Cytomation Denmark A/S) (1-5 min). A Dako Autostainer was used, according to the standard staining procedure. The detection system was Dako Envision Flex+/High pH. The following primary antibodies were used to identify different cell types: MCs: rabbit polyclonal anti cKIT (diluted 1:400); macrophages: mouse monoclonal anti-CD68, IgG1 kappa (diluted 1:5,000); endothelial cells: mouse monoclonal anti-CD31, IgG1 kappa (diluted 1:200); CAFs: mouse monoclonal anti $\alpha$-smooth muscle actin ( $\alpha$-SMA), IgG2a kappa (diluted 1:500) (all from Dako, Glostrup, Denmark); and fibroblast: rabbit monoclonal anti- $\beta$-type platelet-derived growth factor receptor (PDGFR $\beta$ ), IgG (diluted 1:300) (Cell Signaling, Danvers, MA, USA). Negative controls included replacement of primary antibody with mouse myeloma immunoglobulin (same subtype and concentration as the monoclonal antibody) and phosphate-buffered saline. Positive controls were tumors known to express the antigens of interest.

Immunoreactivity scoring. The immunostained slides were evaluated using a semi-quantitative scale by three observers (CGA, MB and SK) with a Nikon eclipse 90i microscope and a Nikon DS-Ri1 camera (Nikon Instruments INC, Melville, NY, USA). For MCs, however, the number of cells in the stroma at the tumor front was scored by counting the $\mathrm{c}-\mathrm{KIT}^{+}$cells in one field of vision at $\times 200$ magnification, after searching for the most pronouncedly stained area. The other markers were grouped into low, moderate and high expression. A consensus score was obtained among the observers in cases of discrepancy.

Tumor budding. Tumor budding was scored as previously described (10). In brief, single cells or clusters of $\leq 5$ cells were classified as buds in pan-keratin (Thermo Scientific, Fremont, CA, USA)-stained sections. The tumors were grouped into high- ( $\geq 5$ buds) and lowbudding ( $<5$ buds). 
Table I. Markers scored in the tumor microenvironment at the invasive front zone, grouped by expression and recurrence. The prognostic value of c-KIT ${ }^{+}$mast cells is highly significant.

\begin{tabular}{|c|c|c|c|c|}
\hline Marker & Positive cells in stroma at tumor front & No. of cases & No. of recurrences & $p$-Value \\
\hline \multirow[t]{2}{*}{ c-KIT, Mast cells $(\mathrm{N}=54)$} & $<15$ Cells & 39 & 14 & 0.005 \\
\hline & $\geq 15$ Cells & 15 & 0 & \\
\hline \multirow[t]{3}{*}{ CD68, Tumor-associated macrophages $(\mathrm{N}=51)$} & Low & 3 & 1 & 0.943 \\
\hline & Moderate & 17 & 4 & \\
\hline & High & 31 & 8 & \\
\hline \multirow[t]{3}{*}{ CD31, Endothelial cells $(\mathrm{N}=59)$} & Low & 12 & 3 & $0.037 *$ \\
\hline & Moderate & 13 & 6 & \\
\hline & High & 34 & 6 & \\
\hline \multirow[t]{3}{*}{$\alpha$-SMA, CAFs $(\mathrm{N}=60)$} & Low & 19 & 5 & 0.889 \\
\hline & Moderate & 13 & 4 & \\
\hline & High & 28 & 6 & \\
\hline \multirow[t]{3}{*}{ PDGFR $\beta$, Fibroblasts $(\mathrm{N}=52)$} & Low & 3 & 0 & 0.294 \\
\hline & Moderate & 7 & 3 & \\
\hline & High & 42 & 10 & \\
\hline
\end{tabular}

*Reflects recurrences in cases with moderate endothelial cell density, but we consider the clinical relevance not to be of interest.

Statistical analyses. Kaplan-Meier survival analysis was performed, and the differences computed by log-rank test (IBM SPSS Statistics, version 21; IBM Corp., Armonk, NY, USA). The endpoints for survival analyses were time to recurrence, time to last follow-up, or time to death. A case was censored if death occurred from diseases other than the index tumor and if patients were free of disease at the last consultation or contact. Cross tabulation, including chisquare tests were performed for testing association between variables. Statistical significance was defined as $p<0.05$.

\section{Patients with HNSCC}

Clinical data and RNA expression analysis. We searched and downloaded the mRNA expression profiles and clinical data of 498 patients with HNSCC from The Cancer Genome Atlas (TCGA) database: https://tcga-data.nci.nih.gov/tcgafiles/ftp_auth/distro_ ftpusers/anonymous/tumor/hnsc/bcr/biotab/clin/. All patients were diagnosed and treated during 1997-2014, and followed-up until September 30, 2014. For detailed tumor sample acquisition, see (11). Briefly, biopsies were collected (from patient diagnosed with HNSCC) at the time of surgical resection. The patients had not received any prior treatment (chemotherapy or radiotherapy) for their disease. Cases were staged according to Seventh Edition the American Joint Committee on Cancer staging system (12). mRNA expression profiles were estimated by normalizing raw counts of mapped RNA-sequences reads to human reference genes, and mRNA levels measured as fragments per kilobase per million mapped reads. Patients without follow up-data, or who died within 2 months, were excluded, and finally 399 patients, 284 (71\%) men and $115(29 \%)$ women, with a median age of 61 years (range $=19$ to 90 years) were included.

\section{Results}

\section{Patients with OSCC}

Morphology. The WHO and IFG grading systems had no prognostic value for disease recurrence. However, the morphological growth score, pattern of invasion, and tumor budding was associated with prognosis, as noted previously (10): tumors with "pushing, well delineated" border had a better prognosis than those with other patterns $(p=0.13)$, and those with "high budding" tumors experienced relapse more often than those with "low-budding" tumors $(p=0.043)$.

Stromal cells. We scored the expression of several markers to identify stromal cells (Table I). Only MC density was highly associated with prognosis (Figures 1 and 2). The density of macrophages (CD68), CAFs ( $\alpha$-SMA) and endothelial cells (CD31) had no prognostic significance (Table I, Figure 3).

MCs at the invasive front. Sufficient tissue was available in 54 cases. After having tested several cut-off values for MC density with regard to time to recurrent disease, we chose to divide the patients into cases with high or low MC density at the invasive front using a cut-off of $15 \mathrm{c}-\mathrm{KIT}^{+}$cells per view-field at $\times 200$ magnification. Whereas 14 out of 39 patients with fewer than $15 \mathrm{c}-\mathrm{KIT}^{+}$cells developed recurrent disease, none of the 15 patients with high MC density did $(p=0.005$, Figures 1 and 2$)$.

MCs, morphology and recurrence. High-budding tumors had lower MC density as 24/28 high budding tumors had $<15$ MCs/field, whereas only four had an increased MC density at the invasive front $(p=0.01)$. High budding/low mast cell density OSCCs relapsed most frequently [10/24 (42\%), $p=0.018]$. MCs were not significantly correlated to histological grading (WHO and IFG) (data not shown).

$M C$ density correlation to other stromal cells and treatment. MC density at the invasive front did not correlate to 


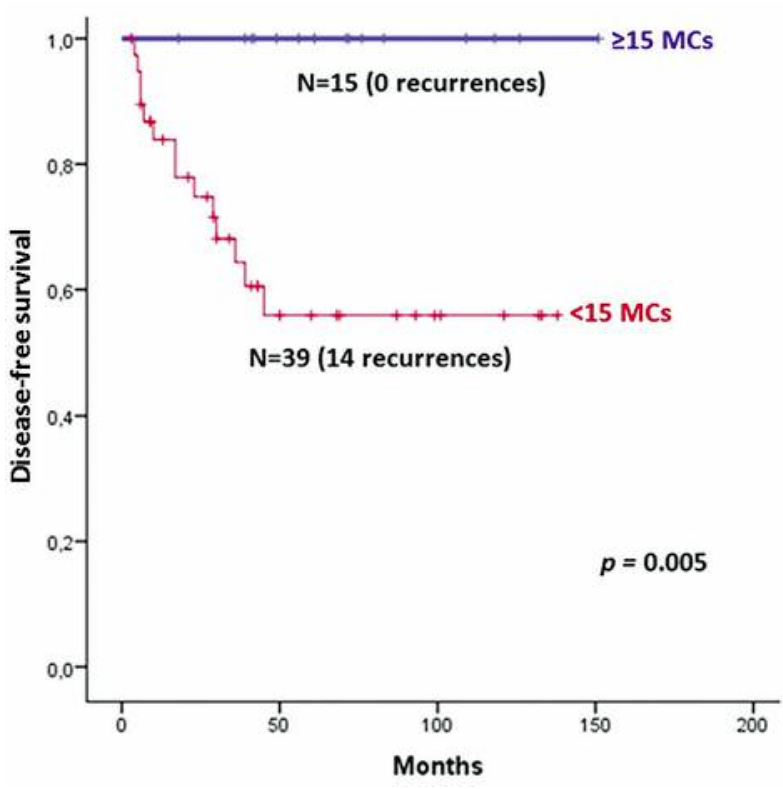

Figure 1. Kaplan-Meier curve of survival according to mast cell (MC) density in the tumor front at $\times 200$.

macrophage $\left(\mathrm{CD} 68^{+}\right), \mathrm{CAF}\left(\alpha-\mathrm{SMA}^{+}\right)$or endothelial $\left(\mathrm{CD} 31^{+}\right)$ cell densities, nor did it influence the treatment's ability to prevent disease recurrence $(p>0.05$, data not shown).

CAFs. $\alpha$-SMA ${ }^{+}$cells primarily surrounded tumor masses and were close to carcinoma cells (Figure 3A and B). All stromal fibroblasts expressed PDGFR $\beta$. Receiving postoperative radiotherapy when having a high CAF density at the invasive front was apparently associated with increased recurrence, as relapse occurred in $28 \%(8 / 29)$ compared with in only one out of 10 irradiated patients with few CAFs (Figure 4A). This was further supported when comparing this to those who had undergone surgery only (Figure 4B): whereas only one of the 11 patients with high CAF density experienced relapse, three out of eight with tumor low CAF density did.

\section{Patients with HNSCC}

c-KIT and genes encoding for MC tryptases in the TCGA database. In order to examine if MC density in HNSCC influenced disease survival, we used the TCGA database. High expression of mRNA for $c$-KIT was associated with better 5year survival (Figure 5A), similar to the reduced disease recurrence revealed by immunhistochemical quantification of MCs at the OSCC invasive front (Figure 1). Expression of $\mathrm{MC}$-specific tryptase-encoding genes were included in the examination. Human genes that encode proteins with tryptase activity include: TPSAB 1 for tryptase $\alpha-1$ and $\beta-1$, and TPSD 1 for $\delta$-tryptase. Low mRNA expression of these genes

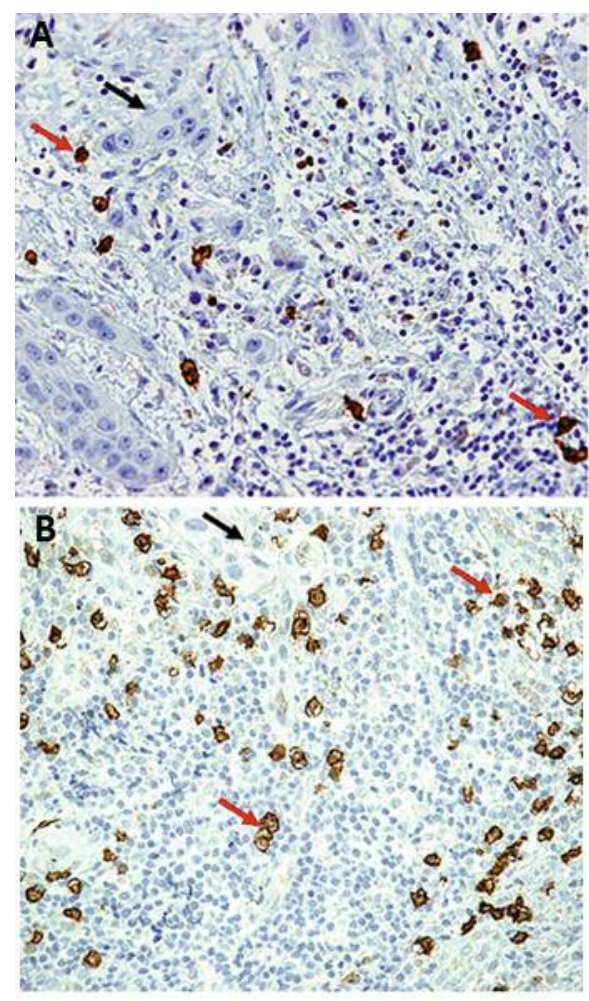

Figure 2. A: Tissue from a patient with low density of mast cells (MCs) (brown cells) in the stroma at the invasive front. B: Tissue from a patient with high density of MCs. Red arrows indicate MCs and black arrows tumor cells (original magnification $\times 200$ ).

predicted poor prognosis $(p<0.01)$ (Figure $5 \mathrm{C}$ and $\mathrm{D})$. $\varepsilon$ Tryptase (encoded by PRSS22), which is not expressed in MCs, although expressed in several cancer types, was not associated with prognosis (data not shown).

mRNA expression for MC-stimulating factors. The mRNA expression of the c-KIT ligand, SCF (KITLG), was associated with increased 5-year survival (Figure 5B), similarly to MCspecific mRNA expression. There was no significant association between thymic stromal lymphopoietin, interleukin $18(I L-18)$ or $I L-33$ mRNA expression and 5-year survival (data not shown).

\section{Discussion}

The biological explanations of how the MC density at the invasive front may influence disease recurrence in patients with OSCC, are unknown. Although secretion of several MC factors has been shown to inhibit tumor growth (13), other mechanisms may also be involved. MCs accumulate in areas when needed, and epithelial cells may produce MC- 

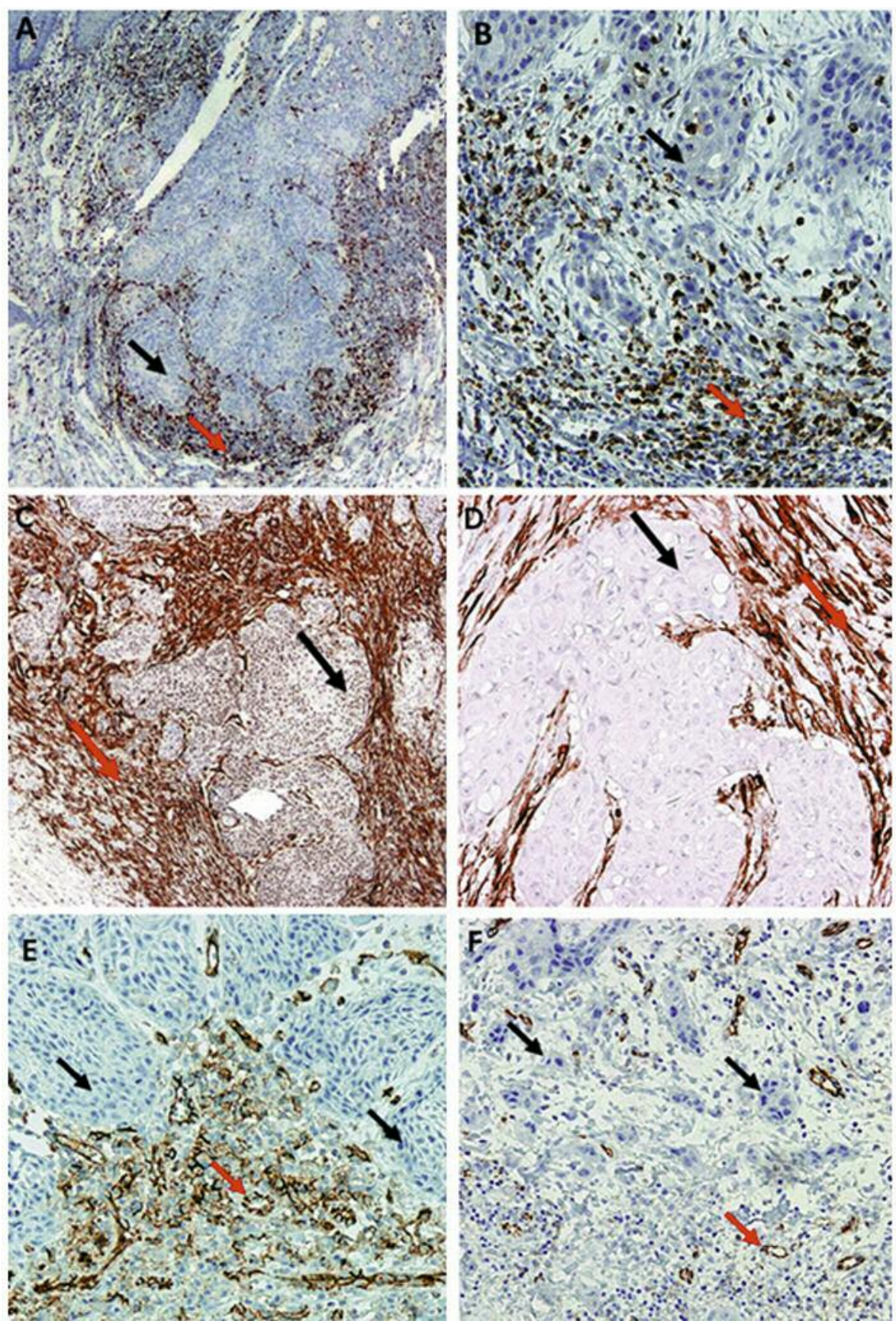

Figure 3. Stromal cells (red arrows) at the invasive front of carcinoma cells (black arrows). A and B: Tumors with a high number of macrophages $\left(C D 68^{+}\right.$, brown cells), $C$ and D: Tumors with high number of cancer-associated fibroblasts ( $\alpha$-smooth muscle actin ${ }^{+}$, brown, elongated cells). E and $F$ : Whereas some patients had a high density of $C D 31^{+}$endothelial cells (brown), often adjacent to cancer cells (E), others had low density (F). Original magnification: $A$ and $C: \times 20, B$ and $D: \times 200, E$ and $F: \times 100$.

stimulating and -attracting factors. It is, therefore, intriguing that expression of MC-specific mRNA encoding c-KIT and tryptases positively correlated with 5-year survival, similarly to mRNA expression of the MC-stimulating c-KIT ligand. Thus, the carcinoma itself may stimulate the presence of MCs.
However, MCs may be the Jekyll and Hyde players in tumor growth because they may both inhibit and stimulate cancer growth $(13,14)$. MCs synthesize and store angiogenic factors and matrix metallo proteinases, that promote tumor vascularization and invasiveness, respectively, and their 

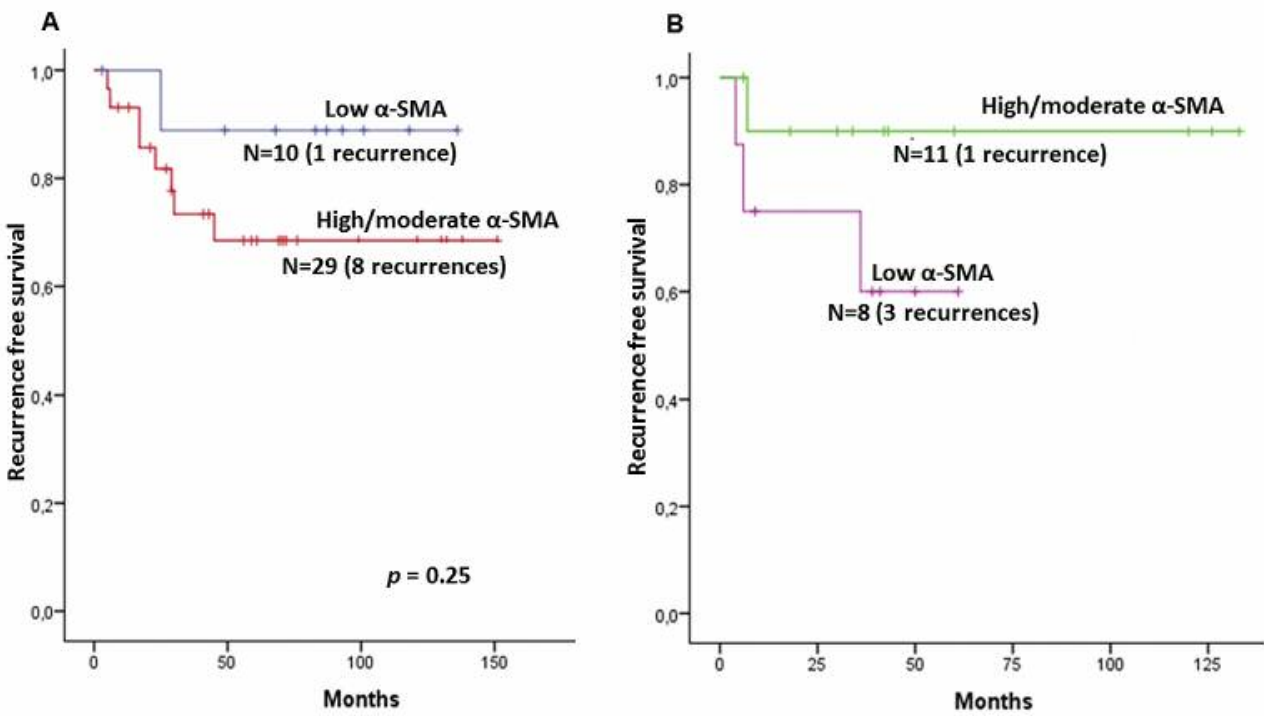

Figure 4. Kaplan-Meier curve of survival according to cancer associated fibroblast (CAF) density $\alpha$-smooth muscle actin ( $\alpha$-SMA) level. A: Among patients who received radiotherapy, there were more relapses in patients with high/moderate CAF density than in those with a low density. B: Among patients who underwent surgery (no radiotherapy), this pattern was reversed, and may reflect a change in irradiated tissue, where CAFs are relatively radioresistant and able to cooperate with residual cancer cells, facilitating tumor relapse.

A

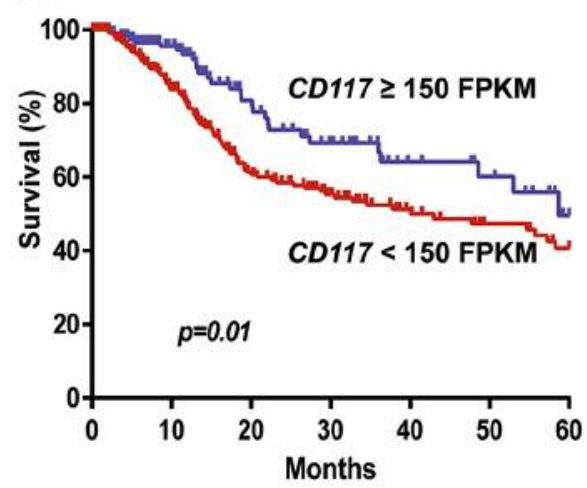

C

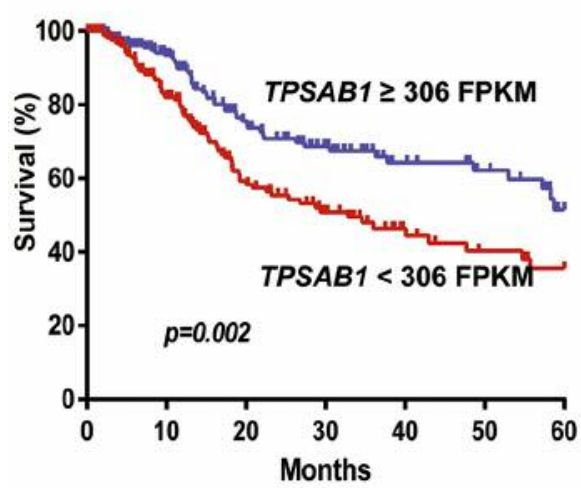

B

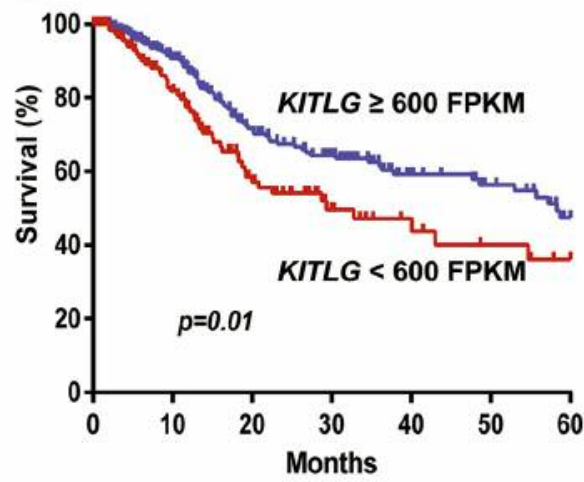

D

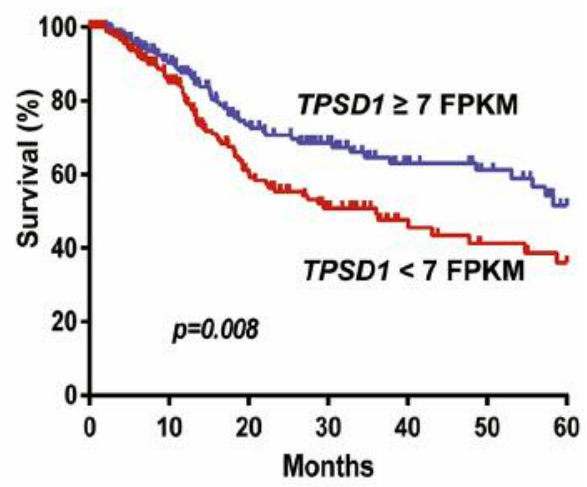

Figure 5. Kaplan-Meier curve of survival of patients with head and neck squamous cell carcinoma (HNSCC) from The Cancer Genome Atlas database according to mast cell (MC)-specific markers. A: Similarly to the immunohistochemically detected c-KIT ${ }^{+}$(CD117) MCs in patients with oral squamous cell carcinoma (see Figure 1), increased c-KIT mRNA expression was significantly associated with increased 5-year survival in patients with HNSCC. B-D: Low expression of MC-specific KIT-ligand (KITLG) (stem cell factor), $\alpha$-, $\beta$ - and $\delta$-tryptases was related to poorer prognosis. FPKM: Fragments per kilobase per million mapped reads. 
histamine release may induce tumor cell proliferation (13). In contrast, MCs may inhibit cancer growth, promote cellular apoptosis and inhibit T-helper cell 1-type of immune response by releasing cytokines such as IL-1, -4 and -6 , transforming growth factor- $\beta$ and tumor necrosis factor- $\alpha$ (13). MC tryptases may induce tumor cell disruption and inflammation through activation of protease-activated receptor-2 (13), which may explain our results. However, increased tryptase secretion may also have adverse effects, such as matrix degradation, which facilitates tumor migration and angiogenesis (15).

Although increased tumoral MC density has been associated with poor prognosis in many studies $(\mathrm{n}=18)(16)$, it has also been associated with good prognosis (16) in cancer, such as esophageal SCC (17), breast (18) and colorectal (19). Moreover, murine epidermal tumors became larger and with more metastasis in c-KIT-deficient mice (20). MC density may be highest initially in tumorigenesis (21), and poorly differentiated tumors had fewer MCs than welldifferentiated OSCCs (22), supporting the concept that SCF from more differentiated OSCCs may stimulate local MC differentiation and therefore be associated with a good prognosis. Reduced MC density in OSCCs with poor prognosis may also reflect microenvironmental changes during squamous epithelial carcinogenesis, making it more permissive to neoplastic cell proliferation (23). Thus, MCs may modulate immune responses, inhibit cancer growth or be a secondary phenomenon related to cancer cell differentiation and production of MC growth factors.

CD68 immunoreactivity occurs with both M1 and M2 macrophages, but the clinical importance of separating macrophages into these subgroups has recently been debated (24). High proportions of TAMs in oral cancer predicted poor clinical outcome (8), perhaps through TAM-induced cancer cell EMT (25), which would facilitate invasive growth and increase metastatic potential. Although we examined TAM density in disease recurrence and not patient survival, macrophage density at the infiltrative front was not prognostically significant.

CAFs may promote carcinogenesis by producing growth, angiogenic, inflammatory and EMT-facilitating factors (6). Increased CAF density is associated with poor prognosis in tongue SCC (26). Although the CAF density did not influence disease recurrence as such, we observed a peculiar tendency for more relapses among patients with a high-CAF density if they received postoperative radiotherapy. Radiotherapy may activate CAFs to produce tumorstimulating factors (27). CAFs survive low-dose (1.8 Gy) but not 3.6 Gy radiation $(28,29)$. The patients of the current study received sequential 2 Gy doses postoperatively, which might not kill CAFs, but merely induce a stress response (29). Such radiotherapy-activated CAFs may, therefore, actually facilitate disease recurrence $e . g$. through increased amphiregulin production (29) or induction of radiotherapy resistance (30). This was also supported by the observation that patients with increased CAF density treated with surgery only had fewer relapses than those with few CAFs. Most patients with more advanced cancer receive pre- or postoperative radiotherapy, and thus, radiotherapy-induced CAF activation may explain why an increased CAF density has been associated with poor prognosis. However, a higher radiation dose, which may kill fibroblasts and CAFs, may also slow wound healing, increase extracellular matrix stiffening and stimulating increased proliferation of surviving cancer cells (28).

Proliferation and metastasis require vascularization, and angiogenesis promotes tumor spread and is associated with poor prognosis (9). Angiogenic factors from cancer cells may induce sprouting and proliferation of endothelial cells (9). However, although there was an increased density of CD31 ${ }^{+}$ endothelial cells in patients with OSCC, this did not correlate with disease recurrence. This is partly in accordance with previous OSCC studies on vessel density, showing diverse prognostic significance $(31,32)$.

\section{Conclusion}

Whereas $36 \%$ of the patients with a low MC density at the OSCC invasive front experienced relapse, none of those with a high MC density in tumors did. A similar association was observed in a cohort patients with HNSCC, where increased mRNA expression of c-KIT, SCF and MC-specific tryptases was significantly associated with increased 5-year survival. A low number of MCs may indicate a need for adjuvant therapy. Thus, MC density at the invading front might have use as a prognostic marker. Counting MCs is easy, and may prove serviceable in clinical practice.

\section{Conflicts of Interest}

None declared.

\section{Acknowledgements}

The Authors thank Ellen Hellesylt for technical support, Jahn M. Nesland for supervision and Hari P. Dhakal for histological grading. (All from Department of Pathology, Radiumhospitalet, Oslo University Hospital).

\section{References}

1 Lim YZ and South AP: Tumour-stroma crosstalk in the development of squamous cell carcinoma. Int J Biochem Cell Biol 53: 450-458, 2014.

2 Hanahan D and Coussens LM: Accessories to the crime: Functions of cells recruited to the tumor microenvironment. Cancer Cell 21(3): 309-322, 2012. 
3 Polyzoidis S, Koletsa T, Panagiotidou S, Ashkan K and Theoharides TC: Mast cells in meningiomas and brain inflammation. J Neuroinflammation 12: 170, 2015.

4 Anuradha A, Kiran Kumar Naik B, Vijay Srinivas G, Devi RS and Puneet HK: Incidence of mast cells in oral squamous cell carcinoma: A short study. J Oncol 2014: 614291, 2014.

5 Galinsky DST and Nechushtan H: Mast cells and cancer - no longer just basic science. Crit Rev Oncol/Hematol 68(2): 115130, 2008.

6 Erez N, Truitt M, Olson P, Arron ST and Hanahan D: Cancerassociated fibroblasts are activated in incipient neoplasia to orchestrate tumor-promoting inflammation in an NF-kappadependent manner. Cancer Cell 17(2): 135-147, 2010.

7 Gwak JM, Jang MH, Kim DI, Seo AN and Park SY: Prognostic value of tumor-associated macrophages according to histologic locations and hormone receptor status in breast cancer. PLoS ONE 10(4): e0125728, 2015.

8 Fujii N, Shomori K, Shiomi T, Nakabayashi M, Takeda C, Ryoke $\mathrm{K}$ and Ito $\mathrm{H}$ : Cancer-associated fibroblasts and CD163positive macrophages in oral squamous cell carcinoma: Their clinicopathological and prognostic significance. J Oral Pathol Med 41(6): 444-451, 2012.

9 Weis SM and Cheresh DA: Tumor angiogenesis: Molecular pathways and therapeutic targets. Nat Med 17(11): 1359-1370, 2011.

10 Attramadal CG, Kumar S, Boysen ME, Dhakal HP, Nesland JM and Bryne M: Tumor budding, EMT and cancer stem cells in T1-2/N0 oral squamous cell carcinomas. Anticancer Res 35(11): 6111-6120, 2015 .

11 Edge SB and Compton CC: The American Joint Committee on Cancer: The 7th edition of the AJCC cancer staging manual and the future of TNM. Ann Surg Oncol 17(6): 1471-1474, 2010.

12 The Cancer Genome Atlas Network: Comprehensive genomic characterization of head and neck squamous cell carcinomas. Nature 517(7536): 576-582, 2015.

13 Ribatti D: Mast cells as therapeutic target in cancer. Eur J Pharmacol 778: 152-157, 2016.

14 Theoharides TC and Conti P: Mast cells: The Jekyll and Hyde of tumor growth. Trends Immunol 25(5): 235-241, 2004.

15 Blair RJ, Meng H, Marchese MJ, Ren S, Schwartz LB, Tonnesen MG and Gruber BL: Human mast cells stimulate vascular tube formation. Tryptase is a novel, potent angiogenic factor. J Clin Invest 99(11): 2691-2700, 1997.

16 Rigoni A, Colombo MP and Pucillo C: The role of mast cells in molding the tumor microenvironment. Cancer Microenviron 8(3): 167-176, 2015.

17 Wang B, Li L, Liao Y, Li J, Yu X, Zhang Y, Xu J, Rao H, Chen S, Zhang L and Zheng L: Mast cells expressing interleukin 17 in the muscularis propria predict a favorable prognosis in esophageal squamous cell carcinoma. Cancer Immunol Immunother 62(10): 1575-1585, 2013.

18 Rajput AB, Turbin DA, Cheang MC, Voduc DK, Leung S, Gelmon KA, Gilks CB and Huntsman DG: Stromal mast cells in invasive breast cancer are a marker of favourable prognosis: A study of 4,444 cases. Breast Cancer Res Treat 107(2): 249257, 2008.

19 Tan SY, Fan Y, Luo HS, Shen ZX, Guo Y and Zhao LJ: Prognostic significance of cell infiltrations of immunosurveillance in colorectal cancer. World J Gastroenterol 11(8): 1210-1214, 2005.
20 Siebenhaar F, Metz M and Maurer M: Mast cells protect from skin tumor development and limit tumor growth during cutaneous de novo carcinogenesis in a kit-dependent mouse model. Exp Dermatol 23(3): 159-164, 2014.

21 de Souza DA, Jr., Toso VD, Campos MR, Lara VS, Oliver C and Jamur MC: Expression of mast cell proteases correlates with mast cell maturation and angiogenesis during tumor progression. PLoS One 7(7): e40790, 2012.

22 Cheema VS, Ramesh V and Balamurali PD: The relevance of mast cells in oral squamous cell carcinoma. J Clin Diagn Res 6(10): 1803-1807, 2012.

23 Oliveira-Neto HH, Leite AF, Costa NL, Alencar RC, Lara VS, Silva TA, Leles CR, Mendonca FE and Batista AC: Decrease in mast cells in oral squamous cell carcinoma: Possible failure in the migration of these cells. Oral Oncol 43(5): 484-490, 2007.

24 Italiani $P$ and Boraschi D: From monocytes to M1/M2 macrophages: Phenotypical vs. functional differentiation. Front Immunol 5: 514, 2014.

$25 \mathrm{Hu}$ Y, He MY, Zhu LF, Yang CC, Zhou ML, Wang Q, Zhang W, Zheng YY, Wang DM, Xu ZQ, Wu YN and Liu LK: Tumorassociated macrophages correlate with the clinicopathological features and poor outcomes via inducing epithelial to mesenchymal transition in oral squamous cell carcinoma. J Exp Clin Cancer Res 35(1): 12, 2016.

26 Bello IO, Vered M, Dayan D, Dobriyan A, Yahalom R, Alanen K, Nieminen P, Kantola S, Laara E and Salo T: Cancer-associated fibroblasts, a parameter of the tumor microenvironment, overcomes carcinoma-associated parameters in the prognosis of patients with mobile tongue cancer. Oral Oncol 47(1): 33-38, 2011.

27 Barcellos-Hoff MH, Park C and Wright EG: Radiation and the microenvironment - tumorigenesis and therapy. Nat Rev Cancer 5(11): 867-875, 2005.

28 Qayyum MA, Kwak JT and Insana MF: Stromal-epithelial responses to fractionated radiotherapy in a breast cancer microenvironment. Cancer Cell Int 15: 67, 2015.

29 Martinez-Zubiaurre I, Fenton CG, Taman H, Pettersen I, Hellevik $\mathrm{T}$ and Paulssen RH: Tumorigenic responses of cancer-associated stromal fibroblasts after ablative radiotherapy: A transcriptomeprofiling study.J Cancer Ther 04(01): 208-250, 2013.

30 Barker HE, Paget JT, Khan AA and Harrington KJ: The tumour microenvironment after radiotherapy: Mechanisms of resistance and recurrence. Nat Rev Cancer 15(7): 409-425, 2015.

31 Carlini MJ, Dalurzo MC, Lastiri JM, Smith DE, Vasallo BC, Puricelli LI and Lauria de Cidre LS: Mast cell phenotypes and microvessels in non-small cell lung cancer and its prognostic significance. Hum Pathol 41(5): 697-705, 2010.

32 Tanaka T, Sho M, Takayama T, Wakatsuki K, Matsumoto S, Migita K, Ito M, Hamada K and Nakajima Y: Endothelin B receptor expression correlates with tumour angiogenesis and prognosis in oesophageal squamous cell carcinoma. Br J Cancer 110(4): 1027-1033, 2014.
Received July 21, 2016

Revised September 2, 2016

Accepted September 20, 2016 